\title{
Comparative Analysis of Wavelet Transforms in the Recognition of Ancient Grantha Script
}

\author{
Jyothi R. L. and Abdul Rahiman M.
}

\begin{abstract}
Wavelet theory is one of the greatest achievements of last decade. The results produced by wavelet based analysis have really astonished the modern research communities in various fields. Wavelet based analysis is still an active research area due to its tremendous variety of applications. This paper provides the comparative analysis of various wavelet transforms to recognize ancient Grantha script. Grantha Script is an ancient script that is used in southern part of India to write Sanskrit language and the motivation of this work is to explore the hidden information from the ancient documents written in Grantha script. For the recognition of ancient Grantha script, a comparative analysis of various transforms like haar, biorthogonal, coiflet, daubechies, discrete meyer and symlet wavelet families are carried out. Discrete meyer wavelet produces the highest recognition efficiency compared to other wavelet families. In this work, the Feed Forward Neural network is used for classification purpose.
\end{abstract}

Index Terms-Biorthogonal, coiflet, daubachies, discrete meyer, grantha script, symlet.

\section{INTRODUCTION}

Manuscripts of ancient India are rich resources for knowledge in astrology, astronomy, vedic mathematics, literature, philosophy. Here an automated character recognition system has been proposed for recognition of Grantha Script found in manuscripts like palm leaves (thaliyolas).

Grantha script is one of the oldest scripts used in Ancient India to write Sanskrit language. Grantha characters consist of 16 vowels, 9 numerals and 34 consonants (Fig. 1).

\section{RELATED WORKS}

L.Huang et al. [1] proposed a new multiresolution recognition scheme for handwritten Chinese character recognition in which an input pattern is recognized by adopting the coefficients of the wavelet transforms. T. T. T. Bui et al [2] proposed a method where combination of wavelet transforms and PCA has been used as character feature for classification. L. Renjini, R. L. Jyothi [3] performed a survey on various types of wavelet transform and its applications. Lee et al [4] proposed a system for recognition of handwritten numerals with coefficients of wavelet transforms are extracted as a multiresolution feature

Manuscript was received on May 20, 2016; revised December 20, 2016. Jyothi. R. L is with College of Engineering, Karunagapally. She is undergoing her Ph.D at Kerala University, India (e-mail: jyothianil@gmail.com)

Abdul Rahiman M. is with Kerala Technological University. He is the research guide under faculty of Engineering in Kerala University, India (email: rahiman_paika@gmail.com). vector by convolving haar wavelets with a character image and multilayer neural network is trained with this multiresolution feature vector. This method enables us to have a scale invariant interpretation of the character image and the details of character image at different resolutions generally characterize different physical structures of the character coefficients obtained from wavelet transform. They are very useful in recognizing totally unconstrained handwritten numerals. Suzete E. N. Correia et al [5] in the paper found an approach for off- line recognition of unconstrained handwritten numerals. This approach uses the Cohen-Daubechies family of biorthogonal spline wavelets as a feature extractor for absorbing local variations in handwritten characters and a multilayer cluster neural network as classifier. The human vision system effortlessly recognizes familiar shapes despite all changes and distortions found in the retinal images. In [6] an approach for recognition of handwritten character was proposed, which is based on human perception. In [7] a method was proposed based on Mexican hat wavelet kernel for license plate character recognition. In [8] a method was proposed based on wavelet energy derived using wavelet transform coefficients for recognition of hand written characters. In [9] a method was proposed based on local binary pattern calculated on the character images decomposed using wavelet transform. In [10] a survey on multiscale image analysis like contourlet, ridgelet, curvelet and their applications was carried out.

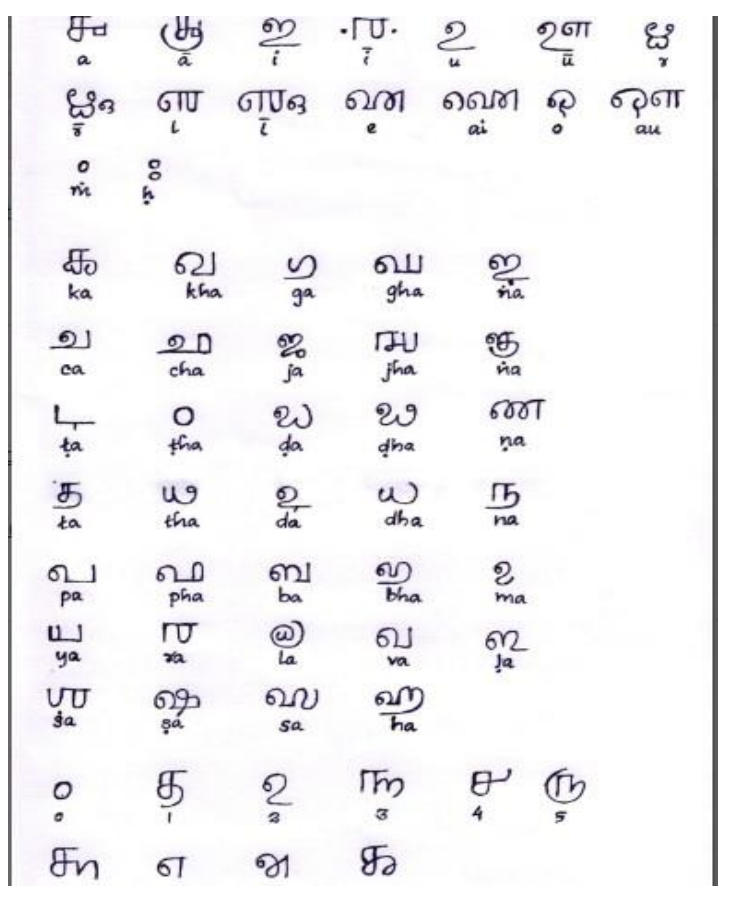

Fig.1. Grantha characters. 


\section{HANDWRITTEN CHARACTER RECOGNITION}

\section{A. Preprocessing:}

The major steps in character preprocessing are image enhancement, noise removal, contrast adjustment, binarization, and morphological operation. In this work binarization is carried out using Otsu's method and thinning is carried out by comparing morphological skeletonization operations and Hilditch algorithm [11]-[13].

\section{B. Feature Extraction:}

In this paper different versions of daubechies, discrete meyer, symlet, coiflet, biorthogonal spline wavelet and reverse biorthogonal wavelets are analyzed and compared for recognition of Ancient Grantha Characters. Wavelet transform [13], [14] allows researchers to manipulate specific types of patterns hidden in data. It performs data analysis from courser to finer details. This transform performs both time and frequency localization and has been developed to overcome the deficiency of fourier transform that performs only frequency localization.

1) Daubechies wavelet:

In Daubachies wavelet system there is no explicit function but the operation is carried out using wavelet and scaling coefficients which forms the low pass and high pass filter coefficients. The scaling function and wavelet function of wavelet transform is given by

$$
\begin{aligned}
& \left.\phi(t)=\sum_{k=0}^{N-1} h(k) \sqrt{2} \phi(2 t-k) \mathrm{k}\right) \\
& \left.\Psi(t)=\sum_{k=0}^{N-1} g(k) \sqrt{2} \phi(2 t-k) \mathrm{k}\right)
\end{aligned}
$$

In this paper 15 versions of daubechies are analyzed for the recognition of grantha characters (db1-db15 or DB2DB20). Up to level 3 of decomposition is carried out and the number of zero crossings [15] and the Principal components of detail part of the wavelet decomposition taken as features for classification.

\section{2) Coiflet:}

Coiflet wavelets are obtained by imposing varnishing moment condition on both scaling and wavelet functions and thereby inducing more coefficients. In this case the minimum number of taps is four. If the number of tap is $\mathrm{N}=6 \mathrm{p}$ then $2 \mathrm{p}$ number of vanishing moments are there in wavelet function and $2 \mathrm{p}-1$ vanishing moments in scaling function.

3) Symlet:

The solutions for wavelet given by Daubechies are not always unique and have maximum smoothness. Based on the intention to induce symmetry to the solutions daubechies induced symlets. The constraints that has been induced into symlets are orthogonal, compact support filter length of $\mathrm{N}=2 \mathrm{p}$.It has $\mathrm{p}$ vanishing moments and it is nearly linear phase.

\section{4) Biorthogonal wavelet system:}

In Biorthogonal systems scaling and wavelet functions are developed based on orthogonality principle in vector space. Consider two square matrices $\mathrm{A}$ and $\mathrm{B}$ let a1, a2.... be the row vectors of matrix $A$ and $b 1, b 2 \ldots$ are row vectors of matrix B. Two matrices are said to be biorthogonal if $\mathrm{i} \neq \mathrm{j}$ $a_{i} \perp b_{j}$ else $a_{i}$ is not perpendicular to $b_{j}$ where $a_{i}$ is not perpendicular to $a_{j}, b_{i}$ is not perpendicular to $b_{j}$ for all $\mathrm{i}$ and j. In case of biorthogonal wavelet system the properties of scaling and wavelet functions are designed based on the property of biorthogonality.

$$
\begin{aligned}
& \int \varnothing(t-k)(\widetilde{\varnothing})(t-m) d t=0 \text { If } \mathrm{k} \neq \mathrm{m}, \\
& \text { otherwise }=1
\end{aligned}
$$

\section{5) Discrete meyer wavelet:}

The Meyer wavelet (dmey) which is an infinitely regular orthogonal and symmetrical wavelet, named after another one of the originators of the field, Yves Meyer. The Haar and Daubechies are compactly supported orthogonal wavelets. These wavelets along with Meyer wavelets are capable of perfect reconstruction. Reconstructed images quality is measured by the peak signal noise ratio, which is obtained by maximum discrete Meyer wavelet. The Meyer wavelet and scaling function are defined in the frequency domain as.

$$
\begin{aligned}
& \psi(\omega)= \\
& \left\{\begin{array}{cc}
\frac{1}{\sqrt{2 \pi}} \operatorname{Sin}\left(\frac{\pi}{2} \mathrm{v}\left(\frac{3|\omega|}{2 \pi}-1\right)\right) \mathrm{e}^{\frac{\mathrm{j} \omega}{2}} \text { if } \frac{2 \pi}{3}<|\omega|<\frac{4 \pi}{3} \\
\frac{1}{\sqrt{2 \pi}} \operatorname{Cos}\left(\frac{\pi}{2} \mathrm{v}\left(\frac{3|\omega|}{2 \pi}-1\right)\right) \mathrm{e}^{\frac{\mathrm{j} \omega}{2}} \text { if } \frac{4 \pi}{3}<|\omega|<\frac{8 \pi}{3} \\
0 & \text { Otherwise }
\end{array}\right.
\end{aligned}
$$

$$
\begin{aligned}
& \emptyset(\omega)= \\
& \left\{\begin{array}{cc}
\frac{1}{\sqrt{2 \pi}} & \text { if }|\omega|<\frac{2 \pi}{3}, \\
\frac{1}{\sqrt{2 \pi}} \operatorname{Cos}\left(\frac{\pi}{2} v\left(\frac{3|\omega|}{2 \pi}-1\right)\right) \text { if } \frac{2 \pi}{3}<|\omega|<\frac{4 \pi}{3} \\
0 & \text { Otherwise }
\end{array}\right.
\end{aligned}
$$

\section{Classification}

Classification is the process of assigning the data to their corresponding class with respect to similar groups with the aim of discriminating multiple objects from each other within the image. The wavelet features extracted from the above phase are trained and tested with feed forward neural network with 150 hidden neurons. Classifier compares input features with stored pattern and find out best matching class of input.

\section{RESUlt AND Discussion}

The experiments are carried out in the folios of the palm leaves taken from Oriental Research Institute, University f Kerala. Grantha characters were extracted from 4,015 folios of ancient palm leaves. Example of a grantha palm leaf is shown in Fig. 2.

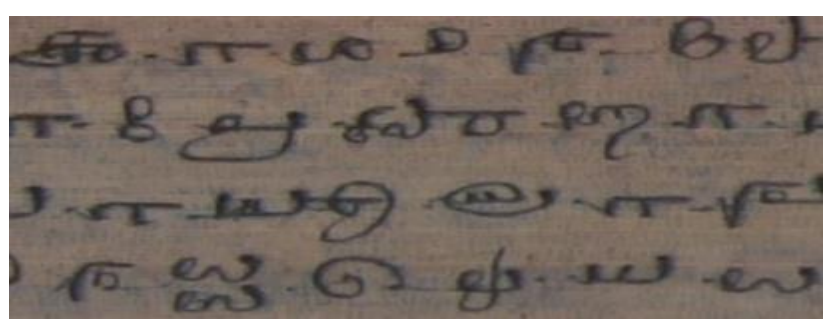

Fig. 2. A palm leaf containing grantha characters. 
Forty two different grantha characters of minimum 290 samples each were selected for training the neural network classifier. The scanned images of the palm leaves are subjected to following steps of processing.

- $\quad$ Binarization using otsu's method[16]

- Thinning using hilditch algorithm[17]

- Segmentation using combination of projection analysis and connected components labeling [18], [19].

- $\quad$ Feature extraction using different family of wavelet transform

Binarization result of a grantha palm leaf when subjected to otsu's binarization is shown in Fig. 3. Thinning result of the binarized grantha palm leaf using Hilditch thinning algorithm is shown in Fig. 4. The thinned result of the source palm leaves are subjected to segmentation using combination of projection analysis and connected components labeling. The touching characters resulted after segmentation using combination of projection analysis and connected components are submitted for further segmentation using drop fall algorithm [20]. Fig. 5 shows the segmentation result of grantha character 'dha'.

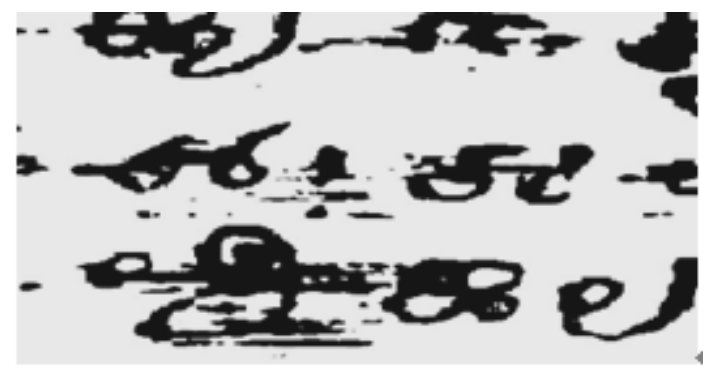

Fig. 3. Binarization result of a sample palm leaf using Otsu's method.

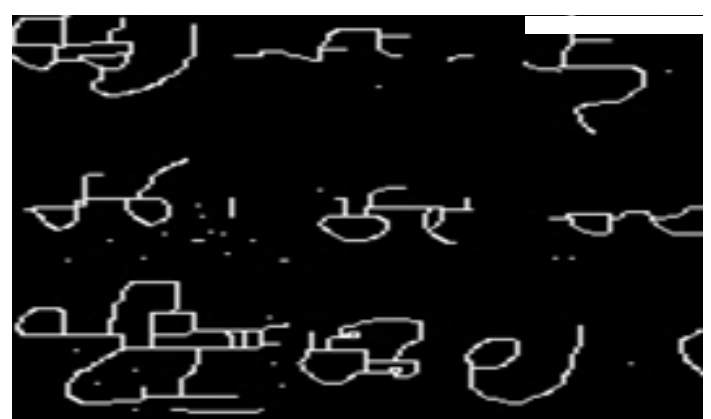

Fig. 4. Thinning result of a sample palm leaf using Hilditch algorithm.

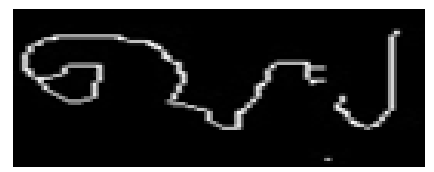

Fig. 5. Segmentation result of grantha character 'dha'.

TABLE I: RECOGNITION EFFICIENCY PLOTTED USING CONFUSION MATRIX FOR 10 CHARACTERS

\begin{tabular}{|c|c|c|}
\hline Feature Vector & Accuracy in \% & Feature length \\
\hline bior1.1 & 94 & 692 \\
\hline bior1.3 & 90 & 756 \\
\hline bior1.5 & 90 & 828 \\
\hline bior2.2 & 90 & 756 \\
\hline bior2.4 & 86 & 828 \\
\hline bior2.6 & 90 & 896 \\
\hline bior2.8 & 86 & 968 \\
\hline
\end{tabular}

\begin{tabular}{|c|c|c|}
\hline bior3.1 & 88 & 732 \\
\hline bior3.3 & 86 & 796 \\
\hline bior3.5 & 88 & 868 \\
\hline bior3.7 & 90 & 936 \\
\hline bior3.9 & 88 & 1008 \\
\hline bior4.4 & 90 & 828 \\
\hline bior5.5 & 92 & 868 \\
\hline bior6.8 & 90 & 968 \\
\hline coif1 & 90 & 756 \\
\hline coif2 & 88 & 868 \\
\hline coif3 & 86 & 968 \\
\hline coif4 & 88 & 1072 \\
\hline coif5 & 90 & 1172 \\
\hline $\mathrm{db} 1$ & 92 & 692 \\
\hline $\mathrm{db} 2$ & 90 & 732 \\
\hline $\mathrm{db} 3$ & 92 & 756 \\
\hline $\mathrm{db} 4$ & 90 & 796 \\
\hline $\mathrm{db} 5$ & 86 & 828 \\
\hline $\mathrm{db} 6$ & 88 & 868 \\
\hline $\mathrm{db} 7$ & 90 & 896 \\
\hline $\mathrm{db} 8$ & 86 & 936 \\
\hline $\mathrm{db} 9$ & 90 & 968 \\
\hline $\mathrm{db} 10$ & 86 & 1008 \\
\hline $\mathrm{db} 11$ & 92 & 1032 \\
\hline $\mathrm{db} 12$ & 92 & 1072 \\
\hline db13 & 90 & 1104 \\
\hline db14 & 88 & 1144 \\
\hline $\mathrm{db} 15$ & 86 & 1172 \\
\hline Haar & 92 & 692 \\
\hline Dmey & 90 & 2412 \\
\hline rbio1.1 & 88 & 692 \\
\hline rbio1.3 & 90 & 756 \\
\hline rbio1.5 & 92 & 828 \\
\hline rbio2.2 & 90 & 756 \\
\hline rbio2.4 & 94 & 828 \\
\hline rbio2.6 & 92 & 896 \\
\hline rbio2.8 & 88 & 968 \\
\hline rbio3.1 & 92 & 732 \\
\hline rbio3.3 & 92 & 796 \\
\hline rbio3.5 & 92 & 868 \\
\hline rbio3.7 & 88 & 936 \\
\hline rbio3.9 & 84 & 1008 \\
\hline rbio4.4 & 88 & 828 \\
\hline rbio5.5 & 86 & 868 \\
\hline rbio6.8 & 92 & 968 \\
\hline sym2 & 90 & 732 \\
\hline sym3 & 92 & 756 \\
\hline sym4 & 90 & 796 \\
\hline sym5 & 88 & 828 \\
\hline sym6 & 90 & 868 \\
\hline sym7 & 86 & 896 \\
\hline sym8 & 88 & 936 \\
\hline sym9 & 88 & 968 \\
\hline sym10 & 84 & 1008 \\
\hline
\end{tabular}

The above methods for preprocessing steps like binarization, thinning and segmentation were selected based on literature analysis. Most of the papers in the area of 
character recognition reported these methods to be efficient for concerned steps in preprocessing. Each resultant isolated grantha character is subjected for feature extraction procedure using different families of wavelet transform. Different families of wavelets arebiorthogonal (bior), coiflet (coif), Daubechies (db), reversebiorthogonal (rbio), haar, Discrete Meyer transform (Dmey) and symlet (Sym). Daubechies wavelet family of versions from db1-db15 are used for feature extraction. The Daubechies wavelet family is characterized into different versions based on the varnishing moment concept. The Daubechies family db1 has one vanishing moment, $\mathrm{db} 2$ has two vanishing moment db3 has 3 vanishing moments and so on. Biorthogonal wavelets of versions bior 1.1 to 6.8 are also used for feature extraction of isolated grantha characters. In case of biorthgonal wavelets each version is denoted by biorx.y where $\mathrm{x}$ indicates the vanishing moments for analysis part (decomposition) and $y$ indicates the vanishing moments for synthesis part (reconstruction).In case of reverse biorthogoanl versions from rbio1.1 to rbio6.8 are used for feature extraction. Various families in reverse biorthogonal wavelets differ in the vanishing moments of analysis and synthesis part as in biorthogonal wavelets. In case of Coiflet (Coif) versions from Coif1-Coif5 are used for analysis. Coiflet wavelet family is characterized into different versions based on vanishing moment concept of wavelet functions and scaling functions. In case of symlets versions sym1-sym5 is used for character analysis. The symlet wavelet is classified into different versions based on the number of vanishing moments for scaling functions. The feature vectors generated as the result of applying different wavelet transforms were fed to ANN classifier for classification. Feed forward neural network with 150 hidden neurons are trained with the feature vectors corresponding to each character. The results analyzed based on confusion matrices generated while applying different families of wavelet transform on grantha characters are shown in Table I, III and V. In Table I recognition efficiency of various wavelet coefficients when neural network is trained with 10 different characters of minimum 290 samples each is demonstrated. From the result analysis it is found that only some of the versions of wavelet families produces efficiency of recognition above 90 percentage All those versions of wavelet transform that has produced recognition efficiency above 90 percentage when tested with 10 different characters of minimum 290 samples each is tested with 20 different characters of minimum 290 samples each and those with the highest efficiency is again tested with 42 different characters with minimum 290 input samples each. From Table I it can be concluded that on considering each family of wavelet transform, some of the versions in each family of wavelet transform tend to produce recognition efficiency greater than 90 percentages. Some of the versions in each family of wavelet transform along with haar and discrete mayer produce recognition efficiency greater than 90. When tested with 10 entirely different characters a particular version in biothogonal and reverse biorthogonal tends to produce the highest recognition efficiency compared to all other wavelet families. The average recognition efficiency of each class of wavelet family when tested with 10 characters is shown in Table II and illustrated in Fig. 6.
Table III shows the recognition accuracy of selected versions of wavelet transforms when the neural network is trained with wavelet coefficients of 20 characters with 290 samples each. Based on analysis of Table III it can be seen only various versions in three of the wavelet families produces recognition efficiency of 80 percentage and above. The three concerned wavelet families are Biorthogonal, Coiflet and Discrete meyer. In biorthogonal family only 3 versions produce recognition efficiency above 90 percentage and in coiflet one version. These versions in the corresponding wavelet families were chosen for training and testing with more number of characters.

TABLE II: AVERAGE RECOGNITION ACCURACY USING 10 ENTIRELY DIFFERENT CHARACTERS OF MINIMUM 290 SAMPLES EACH

\begin{tabular}{|c|c|}
\hline Feature vector & $\begin{array}{c}\text { Average } \\
\text { RecognitionAccuracy in \% }\end{array}$ \\
\hline Biorthogonal(Biortho) & 89.2 \\
\hline Coiflet(Coeff) & 88.4 \\
\hline Daubechies(Db) & 89.2 \\
\hline Symlet(Sym) & 88.44444 \\
\hline $\begin{array}{c}\text { Reverse } \\
\text { Biorthogonal(Rbio) }\end{array}$ & 89.86667 \\
\hline Haar & 92 \\
\hline $\begin{array}{c}\text { Discrete } \\
\text { Meyer(Dmey) }\end{array}$ & 90 \\
\hline
\end{tabular}

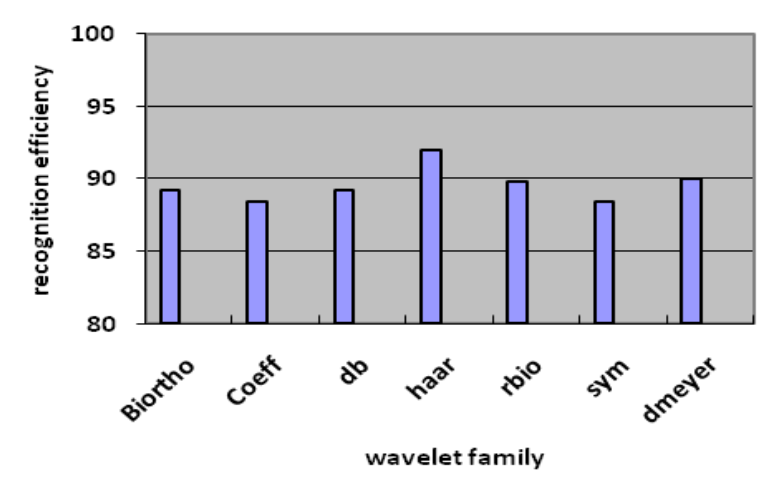

Fig. 6. Response of different wavelet transforms for 10 characters of minimum 290 samples each.

Table $\mathrm{V}$ shows the recognition efficiency of selected wavelet family versions when neural networks were trained with 42 characters of minimum 290 samples each.From the analyzed results it can be seen that discrete meyer wavelet transform produce the highest recognition efficiency. The size of the feature vector that has been derived for classification for each wavelet based method is shown in the concerned tables. As the size of feature vector in case of discrete mayer transform is larger compared to other wavelet families the time taken for classification of discrete meyer transform is slightly higher compared to other wavelet families. But the recognition efficiency of discrete meyer transform feature vectors are very larger compared to other wavelet transforms which overrides its inefficiency of time for recognition. 
TABLE III: RECOGNITION EFFICIENCY PLOTTED USING CONFUSION

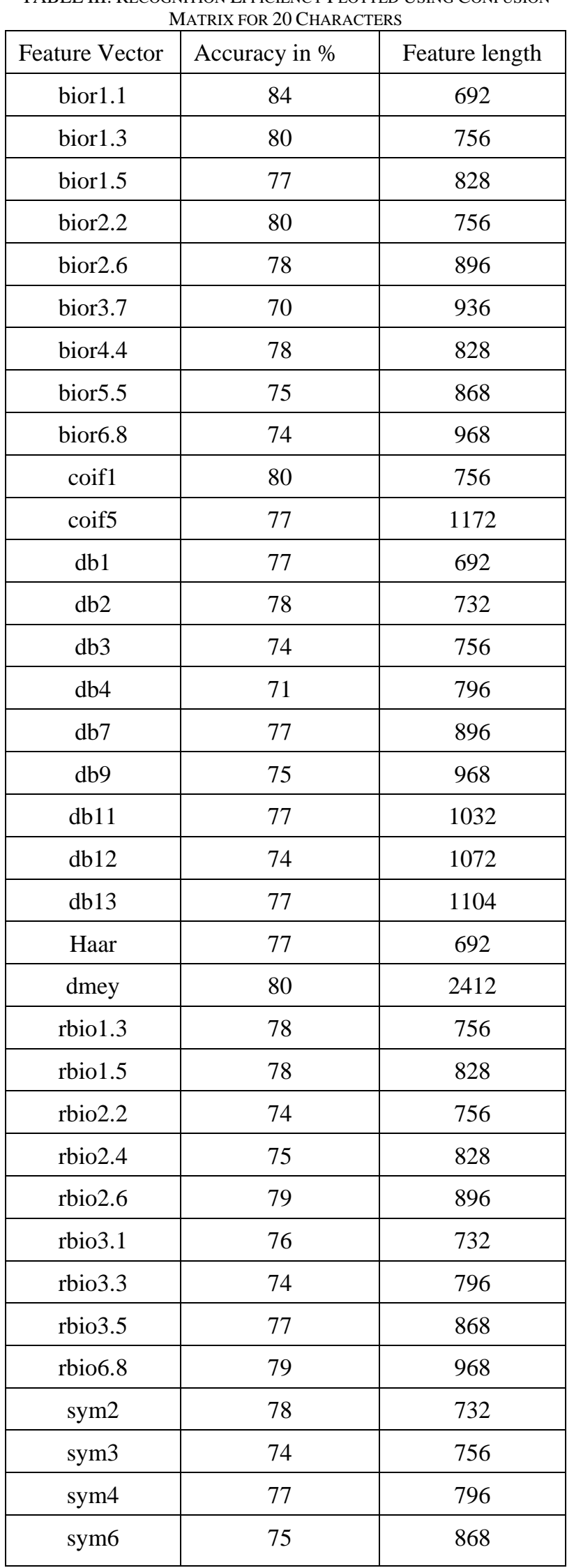

The average response for each wavelet family is shown in Fig. 6, Fig. 7 and Fig. 8. The average response of different wavelet families for 10 characters of minimum 290 samples each is shown in Fig. 6 and Table II. The average response of different wavelet families for 20 characters of minimum 290 samples each is shown in Fig. 7 and Table IV.
TABLE IV: AVERAGE RECOGNITION ACCURACY USING 20 ENTIRELY DIFFERENT CHARACTERS OF MINIMUM 290 SAMPLES EACH

\begin{tabular}{|c|c|}
\hline Feature vector & $\begin{array}{c}\text { Average } \\
\text { RecognitionAccuracy } \\
\text { in } \%\end{array}$ \\
\hline Biorthogonal(Biortho) & 77.33333 \\
\hline Coiflet(Coeff) & 78.5 \\
\hline Daubechies(Db) & 75.55556 \\
\hline Symlet(Sym) & 88.44444 \\
\hline Reverse & 76.66667 \\
\hline Biorthogonal(Rbio) & 77 \\
\hline Haar & 80 \\
\hline Discrete & \\
\hline
\end{tabular}

Response of different wavelet families for 42 characters of minimum 290 samples each is shown in Fig. 7 and Table VI. As the methods which shows lower recognition efficiency is eliminated for each further iteration of classification, last classification iteration with 42 characters were experimented only with three wavelet family (Table III).

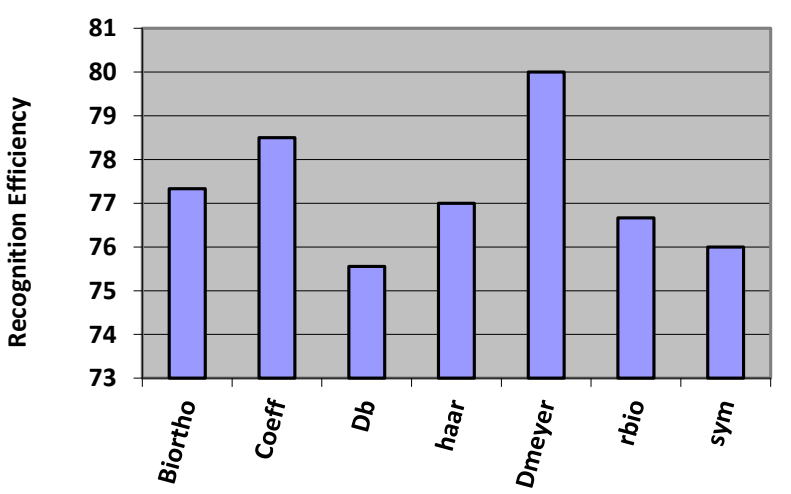

Wavelet family

Fig.7. Average response of various wavelet transform for 20 different characters with minimum 290 samples each.

When trained with 10 entirely different characters and 20 entirely different characters, biorthogonal wavelet transform shows higher recognition efficiency compared to decrete meyer transform but as the number of characters increased to 42 discrete meyer transform shows the highest recognition efficiency. When the number of characters are increased to 42 , more characters with similar almost geometry are included. From the analyzed it can be concluded when there is a large difference in the structure of patterns to be recognised, feature vectors of some of the versions in almost all wavelet families prooves to efficient in recognition by classifiers. As the similarity between characters increases some of the wavelet families overide other. Discrete Meyer, Biorthogonal and reverse biorthogonal prooves to be better compared to other wavelet families when the structure similarities of character 
increases. But when there is a high similarity between characters discrete meyer transform overrides all other wavelet families.Therefore from the analyzed results it can be concluded that decrete meyer tranform is efficient compared to other wavelet transform in analyzing and differentiating very minute changes in the image.Discrete meyer wavelet transform will be very useful in recognition and differentiation of highly complex images.

TABLE V: ReCOGNITION EFFICIENCY PLOTTED USING CONFUSION MATRIX FOR 42 CHARACTERS

\begin{tabular}{|c|c|c|}
\hline \multicolumn{1}{|c|}{$\begin{array}{c}\text { Feature } \\
\text { vector }\end{array}$} & $\begin{array}{c}\text { Accuracy } \\
\text { in } \%\end{array}$ & $\begin{array}{c}\text { Feature } \\
\text { length }\end{array}$ \\
\hline bior1.1 & 48 & 692 \\
\hline bior1.3 & 55 & 756 \\
\hline bior2.2 & 54 & 756 \\
\hline coif1 & 51 & 756 \\
\hline dmey & 71 & 2412 \\
\hline
\end{tabular}

TABLE VI: AVERAGE RECOGNITION ACCURACY USING 42 ENTIRELY DIFFERENT CHARACTERS OF MINIMUM 290 SAMPLES EACH.

\begin{tabular}{|c|c|}
\hline Feature vector & Accuracy in $\%$ \\
\hline bior1.1 & 52.33333 \\
\hline coif 1 & 51 \\
\hline dmey & 71 \\
\hline
\end{tabular}

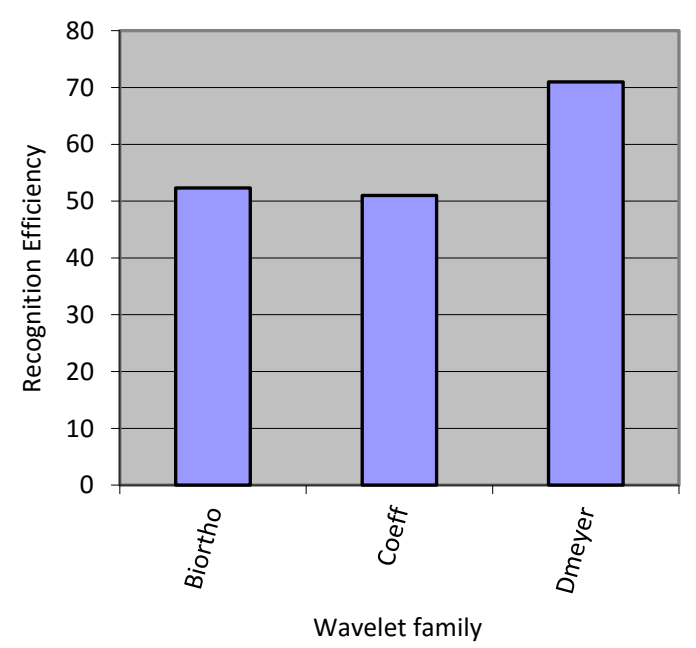

Fig. 8. Average response of various wavelet transform for 42 different characters with minimum 290 samples each.

\section{CONCLUSION}

Wavelet families such as haar, daubechies, coiflet, symlet, discrete meyer, biorthogonal and reverse biorthogonal wavelets are analyzed and compared in recognition of grantha script characters. Forty two grantha characters of minimum 290 input samples each is used for training the classifier. From the analysis it has been found that discrete meyer wavelet basis produces the best classification accuracy of $71 \%$ compared to other wavelet transforms. From the analyzed results it can be concluded that decrete meyer tranform is efficient compared to other wavelet transform in analyzing and differentiating very minute changes in the image.The results produced in this work will be highly useful to the pattern recognition community. This work can be extended to all other areas in pattern recognition.

\section{REFERENCES}

[1] L. Huang and X. Huang. "Mu1tiresolution recognition of offline handwritten Chinese characters with wavelet transform," in Proc. Sixth International Conference on Document Analysis and Recognition, Washington, DC, USA, pp. 631-634, Sept. 2001.

[2] T. T. T. Bui, N. H. Phan, V. G. Spitsyn, Y. A. Bolotova, and Y. V. Savitsky, "Development of algorithms for face and character recognition based on wavelet transforms, PCA and neural networks," The 2015 International Siberian Conference on Control and Communications (Sibcon), pp. 21-23, May 2015.

[3] L. Renjini and R. L. Jyothi, "Wavelet based image analysis: A comprehensive survey," International Journal of Computer Trends and Technology (IJCTT), vol. 21, no. 3, pp. 134-140, Mar. 2015.

[4] S. W. Lee and Y. J. Kim. "Recognition of handwritten numerals with wavelet transform and multilayer cluster neural network," in Proc. Third International Conference of Document Analysis and Recognition, vol. 2, pp.1010-1013, Aug. 1995.

[5] S. E. N. Correia and J. M. D. Carvalho "Optimizing the recognition rates of unconstrained handwritten numerals using biorthogonal spline wavelet," in Proc. 15th International Conference of Pattern Recognition on Barcelona, vol. 2, pp. 251-254, 2000.

[6] S. E. N. Correia, J. M. de. Carvalho, and R. Sabourin. "Humanperception handwritten character recognition using wavelets," in Proc. $X V$ Brazilian Symposium on Computer Graphics and Image Processing, Fortaleza, Brazil, pp. 197-2002, October 2002.

[7] G. Yang, "License plate character recognition based on wavelet

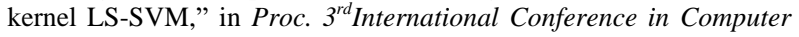
Research and Development, Shanghai, pp. 222-226, May 2011.

[8] B. P. Chacko, V. Krishnan, and G. Raju, "Handwritten character recognition using wavelet energy and extreme learning machine," International Journal of Machine Learning and Cybernetics, vol. 3, no. 2, pp 149-161, June 2012.

[9] A. Mohamed and R. V. Yampolskiy, "Face recognition based on wavelet transform and adaptive local binary pattern," in Proc. International Conference on Digital Forensics and Cyber Crime, pp. 158-166, 2012.

[10] R. S. SuryaNath and A. Anilkumar, "Study on multiscale image analysis: Theory and applications," International Journal of Computer Trends and Technology, vol. 22, no. 1, April 2015.

[11] N. Arica and F. T. Yarman-Vural, "An overview of character recognition focused on off-line handwriting," IEEE Trans on Systems, Man, and Cybernetics, vol. 31, no. 2, pp. 216-233, May 2001.

[12] R. Plamondon, S. N. Srihari. "On-Line and off-line handwriting recognition: A comprehensive survey," IEEE Trans on Pattern Analysis and Machine Intelligence, vol. 22, no.1, pp. 63-84, Jan. 2000.

[13] M. Cheriet, N. Kharma, C. L. Liu, and C. Y. Suen, Character Recognition Systems, A Guide for Students and Practioners, Wiley. Dec. 2007, ch. 3.

[14] R. C. Gonzalez and R. E. Wood, Digital Image Processing, New York: Addison-Wesley, 1992, ch. 7.

[15] S. Mallat, "Zero crossings of a wavelet transform," IEEE Transactions on Information Theory, vol. 37, no. 4, pp. 1019-1033, July 1991.

[16] N. Otsu, "A threshold selection method from graylevel histograms," IEEE Trans on System Man, and Cybernetics, vol. 9, no.1, pp. 62-66, 1979.

[17] Lam, SW Lee, and C. Y. Suen, "Thinning methodologies a comprehensive survey," IEEE Trans on Pattern Analysis and Machine Intelligence, vol. 14, no. 9, pp. 869-885, Sept. 1992.

[18] Z. Abderaouf, B. Nadjia, and O. K. Saliha, "License plate character segmentation based on horizontal projection and connected 
component analysis," in Proc. Symposium on Computer Applications \& Research (WSCAR), pp.18-20, 2014.

[19] S. C. Vijayan, R. L. Jyothy, and A. Anilkumar, "Histogram based connected component analysis for character segmentation," in International Journal of Scientific and Research Publications, vol. 6 , no. 6, pp. 2250-3153, June 2016.

[20] Z. H. Cao, N. China, M. H. Huang, and Y. Wang, "A new dropfalling algorithms segmentation touching character," IEEE International Conference on Software Engineering and Service Sciences, Beijing, pp. 380-383, 2010.

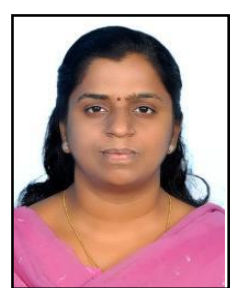

Jyothi R. L. was born on Feb. 19, 1981, received her B Tech degree from St. Xavier's Catholic College of Engineering, Nagercoil, India in the year 2003 and M Tech degree from Anna University, Chennai, India in the year 2008. She is having 13 years of experience currently working as assistant professor in computer science and engineering, College of Engineering Karunagappally. Kerala, India. Her research interests include image processing, pattern recognition and machine learning. She has published several papers in the area of image processing and character recognition.

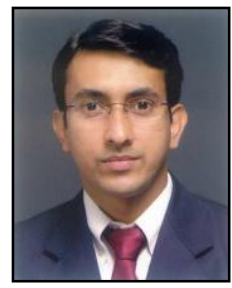

Abdul Rahiman is an eminent academician and an able administrator. He is the Pro Vice chancellor of Kerala Technological University, since September 2014. He received the doctor of philosophy (Ph.D.) degree in computer science \& engineering from Karpagam University. He obtained his master of technology from Kerala University in 2004, and bachelor of technology from Calicut University 1998. He achieved post graduate diploma in human resource management from Kerala University in 2006 \& master of business administration (MBA) in 2008. His specialization is in digital image processing \& pattern recognition and has taught for more than 15 years having a rich teaching experience and guiding many Ph.D scholars in the area of DIP, data mining and networking. He has many international publications into his credit. 